\title{
VULNERABLES Y EMPODERADAS A LA \\ HORA DE PARIR. ANÁLISIS \\ MULTIDIMENSIONAL DE LA \\ VULNERABILIDAD EN LA ATENCIÓN \\ OBSTÉTRICA ${ }^{1}$
}

Vulnerable and Empowered in Childbirth. Multidimensional Analysis of Vulnerability in Obstetric Care

Belén Castrillo ${ }^{1}$

Correo electrónico: mbcastrillo@gmail.com

1. Becaria posdoctoral del CONICET. Centro Interdisciplinario de Metodología de las Ciencias Sociales. Instituto de Investigaciones en Humanidades y Ciencias Sociales. Facultad de Humanidades y Ciencias de la Educación de la Universidad Nacional de La Plata. Dra. en Ciencias Sociales y Lic. en Sociología. (La Plata, Argentina)

Recibido: 14/04/2020 Aceptado: 15/06/2020

\footnotetext{
${ }^{1}$ Parte de los datos de campo aquí citados emergen del trabajo doctoral publicado en la tesis de 2019 de la misma autora citada en bibliografía. Dicha tesis doctoral fue financiada con una beca tipo A de la UNLP y luego una beca de finalización de doctorado del CONICET.
} 


\section{RESUMEN}

OBJETIVO. Tomando en cuenta los dos sentidos clásicos de la vulnerabilidad en ciencias sociales —el antropológico y el social-, el propósito de este artículo es introducir la conceptualización butleriana de la vulnerabilidad —entendida como acto de poder y resistencia - como herramienta teóricometodológica clave para entender y analizar las experiencias perinatales de las mujeres. MATERIAL $\mathbf{Y}$ MÉTODO. La tesis de referencia analizó diversas instancias de la atención médica del proceso perinatal en la ciudad de La Plata, con técnicas metodológicas complementarias: observaciones con distintos grados de participación en cursos de preparto y salas de espera; entrevistas en profundidad a mujeresmadres, varones-padres y profesionales de la salud; y un extenso corpus de charlas informales y presencias en el campo, entre 2013 y 2019. RESULTADOS. En el trabajo de reinterpretación del corpus de datos, se advirtieron tres sentidos principales en que emergió la vulnerabilidad de las mujeres asociadas a la atención obstétrica de sus procesos reproductivos: a) Vulnerabilidad frente a la decisión médica (pérdida de autonomía); b) Vulnerabilidad potenciada por otras vulnerabilidades asociadas; c) Vulnerabilidad y resistencia, empoderamiento. CONCLUSIONES. A partir del cruce entre una lente conceptual novedosa para entender la vulnerabilidad, analicé experiencias de partos de mujeres platenses. Advertí que en estas experiencias y teniendo en cuenta sus propias evaluaciones de atención en distintos ámbitos — público, privado o domicilio— es posible rastrear tres tipos de vulnerabilidad: a) a la decisión médica — pérdida de autonomía—; b) potenciada por otras vulnerabilidades; y, c) como catalizadora del empoderamiento.

Palabras claves: parto; vulnerabilidad; empoderamiento; atención médica.

\section{ABSTRACT}

OBJECTIVE. Taking into account the two classic senses of vulnerability in the social sciences-the anthropological and the social-the purpose of this article is to introduce the Butlerian conceptualization of vulnerability — understood as an act of power and resistance - as a key theoretical-methodological tool for understanding and analyzing women's perinatal experiences. MATERIAL AND METHOD. The reference thesis analyzed various instances of medical care of the perinatal process in the city of La Plata, with complementary methodological techniques: observations with different degrees of participation in prepartum courses and waiting rooms; in-depth interviews with women-mothers, men-fathers, and health professionals; and an extensive corpus of informal talks and presences in the field, between 2013 and 2019. RESULTS. In the reinterpretation of the data corpus, three main senses were noted in which the vulnerability of women associated with obstetric care of their reproductive processes emerged: a) Vulnerability to medical decision (loss of autonomy); b) Vulnerability enhanced by other associated vulnerabilities; c) Vulnerability and resistance, empowerment. ConCLUSIONS. Starting from the cross between a new conceptual lens to understand vulnerability, I analyzed birth experiences of women from La Plata. I warned that in these experiences and taking into account their own evaluations of care in different areas -public, private or home-it is possible to trace three types of vulnerability: a) to the medical decision-loss of autonomy-; b) powered by other vulnerabilities; and, c) as a catalyst for empowerment.

Key words: Childbirth; Vulnerability; Empowerment; Medical Care. 


\section{INTRODUCCIÓN}

Cierta idea establecida y naturalizada a nivel popular sostiene que no hay mayor momento de vulnerabilidad para una mujer que el tránsito por el proceso perinatal. No es mi intención negar esta creencia, sino señalar la posibilidad de que lo que sentencia esa idea esconda la concepción de las mujeres como incapaces para parir y la necesidad de protección e intervención médica, y que postule su subalternización como inherente al proceso. ¿Que la institución médica mantenga el estatuto de vulnerables de las pacientes para justificar su intervención no es condición de su poder? Desde el activismo feminista, en una actitud de revalorización del estigma, se postula que no es vulnerabilidad, sino el germen del empoderamiento silenciado culturalmente, lo que sucede durante el proceso de embarazo, parto y puerperio (PEPP). Así, el lema de la militancia por el parto respetado, «el poder de parir está en vos», se tensa con la doctrina de necesidad médica, en una disputa cuyo trasfondo implica la concepción sexual o médica del parto, la medicalización y patologización de los procesos reproductivos y de la salud de las mujeres (Esteban, 2001; Vals Llobet, 2009; Castrillo, 2019).

Interpelada a pensar la vulnerabilidad de las mujeres en los PEPP y su atención médica, escribo este artículo con el objetivo de reflexionar en qué sentidos al momento de parir las mujeres somos vulnerables o empoderadas. La construcción maniquea de estas dos situaciones como extremos incompatibles no permite, sin embargo, visualizar la convivencia contradictoria y tensa entre espacios e intersticios de vulnerabilidad y empoderamiento conjuntos.

Así, y a raíz de los resultados de la tesis doctoral (Castrillo, 2019) en la que investigué experiencias de mujeres-madres, varones-padres y profesionales de la salud obstétrica sobre intervenciones médicas en los PEPP en la ciudad de La Plata — capital de la provincia de Buenos Aires- entre 2013 y 2019, propongo leer dichos datos con una lente multidimensional sobre la vulnerabilidad.

\section{LA MULTIDIMENSIONALIDAD DE LA VULNERABILIDAD}

En la reconstrucción teórica realizada — que no pretende ser totalizante—, he advertido tres sentidos principales en que se ha pensado la vulnerabilidad y que me permiten 
interpretar mi objeto de estudio. Primero, un sentido ontológico; segundo, un sentido social; y, tercero, y lo que apunta a ser el aporte de este trabajo, la combinación con el sentido aportado por Butler (2015; 2017), quien entiende a la vulnerabilidad como acto de poder, vinculada a acciones de resistencias. Son comunes a los estudios sociológicos las conceptualizaciones de los primeros dos, ${ }^{2}$ tal como se resume en el siguiente fragmento:

Dos tipos de vulnerabilidad humana: una vulnerabilidad antropológica, entendida como una condición de fragilidad propia e intrínseca al ser humano, por su ser biológico y psíquico; y una vulnerabilidad socio-política, entendida como la que se deriva de la pertenencia a un grupo, género, localidad, medio, condición socio-económica, cultura o ambiente que convierte en vulnerables a los individuos. (Feito, 2007, p. 8)

Tomando en cuenta estos dos sentidos, el propósito de este artículo es introducir la conceptualización butleriana de la vulnerabilidad como herramienta teóricometodológica clave para entender y analizar las experiencias perinatales de las mujeres. Se trata de una teorización que supone las siguientes ideas claves: la vulnerabilidad como posibilidad de resistencia o acto de poder; no como disposición subjetiva sino relacional; como una región intermedia entre ser afectado/a y actuar; vinculada fundamentalmente a la performatividad de género y a cierta política de la desigualdad, a partir de lo que ella entiende como una «distribución desigual de la vulnerabilidad». Nuclearmente, su propuesta busca denunciar el peligro de los usos dominantes de la vulnerabilidad para etiquetar poblaciones y así justificar el paternalismo y el colonialismo, o la pasividad en los sectores marginados y construidos como vulnerables.

La vulnerabilidad no es una disposición subjetiva, sino una relación con un campo de objetos, fuerzas y pasiones que inciden o nos afectan de alguna manera [...]. La vulnerabilidad no es plenamente pasiva ni plenamente activa sino que opera en una región intermedia, lo que es una característica constitutiva del animal humano que es capaz tanto de ser afectado como de actuar. (Butler, 2015, p. 16)

2 Es clásica la teorización de Castel (1995), quien ha entendido este segundo sentido de la vulnerabilidad como «una zona de turbulencias caracterizada por una precariedad en relación al trabajo y por una fragilidad de soportes relacionales» (p. 1). 
La propuesta del artículo es pensar cómo complejizar una mirada que puede ver en las mujeres «objetos de vulnerabilidad» que precisan de cuidado, control y vigilancia, con otra que sugiere que desde la misma vulnerabilidad se pueden producir «sujetos vulnerables» con capacidad de agencia. Para ello retomo la teoría de Judith Butler sobre la vulnerabilidad, reconstruyendo su argumentación a partir de dos trabajos recientes (2015; 2017) con el objetivo de considerar cómo las distintas dimensiones de análisis que propone para abordar la vulnerabilidad se vuelven herramientas clave para interrogar mi propio objeto de estudio: la atención médica de PEPP.

La premisa principal de la idea butleriana sostiene que «la vulnerabilidad, entendida como una exposición deliberada ante el poder, es parte del mismo significado de la resistencia política como acto corporal» (2015, p. 11). En este sentido, se explaya y sostiene que

la resistencia política se basa, fundamentalmente, en la movilización de la vulnerabilidad y que las formas plurales o colectivas de resistencia están estructuradas de forma muy distinta a la idea de un sujeto político que establece su agentividad venciendo su vulnerabilidad; entiendo esto último como un ideal masculinista. (p. 14)

Para complejizar esta mirada, la autora alerta que «los grupos dominantes puedan usar el discurso de la "vulnerabilidad” para apuntalar sus propios privilegios» (p. 13). Y en este sentido sostiene que la etiqueta de «población vulnerable» generada por dichos grupos refuerza la sumisión y la pasividad, en tanto que

hay quienes arguyen que la vulnerabilidad no puede ser la base para una identificación grupal sin que eso refuerce el poder paternalista. Una vez que los grupos son etiquetados como «vulnerables» dentro del discurso de los derechos humanos o los regímenes legales, esos grupos acaban siendo reificados, devienen «vulnerables» por definición, quedan fijados en una posición de indefensión y falta de agentividad. Todo el poder pertenece al estado y las instituciones internacionales que a día de hoy se supone que han de ofrecerles protección y apoyo. (p. 14)

Sin embargo, a esto es preciso sumar que es posible visibilizar un uso político e instrumental de la vulnerabilidad, y no solo en manos de los poderosos, sino, también, como instrumento de resistencia de las mujeres para exigir protección estatal, derechos 
específicos, recursos y beneficios. En este punto, entonces, la vulnerabilidad actúa como instrumento de poder emancipador en manos de mujeres que exigen regímenes especiales frente a la pretendida situación precaria reproductiva. Si somos consideradas vulnerables, incapaces y requeridas de control y vigilancia médica, usémoslo a nuestro favor y en esos mismos términos, exijamos de qué modos seremos medicalizadas, institucionalizadas o cuidadas. En ese marco, la agenda pública y legislativa en Argentina en torno a los derechos sexuales y reproductivos ha tenido como momentos clave en la búsqueda de emancipación la sanción de dos leyes que protegen los derechos humanos de las mujeres en la atención al proceso de parto: la ley 25.929 de 2004, conocida como «Ley de parto respetado», y la ley 26.485 de 2009, conocida como «Ley de violencia de género».

La argumentación butleriana «toma la vulnerabilidad y la invulnerabilidad como efectos políticos, efectos desigualmente distribuidos de un campo de poder que actúa sobre y a través de los cuerpos» (2017, p. 18). Y en este sentido, atraviesa la lectura considerando cómo la desigualdad de vulnerabilidades opera genéricamente.

Algunos atributos para definir los géneros, como vulnerabilidad e invulnerabilidad, son distribuidos de manera desigual desde ciertos regímenes de poder, y precisamente con el propósito de reforzar esos regímenes que privan de derechos a las mujeres. (p. 17-18)

Para mi caso de estudio, en la tesis y a partir del análisis de variados ámbitos sanitarios y de experiencias diversas de atención obstétrica — como se verá en el apartado de los resultados_- he podido advertir cómo la interseccionalidad de vulnerabilidades o de desigualdades — de clase, de edad, de etnia — le aporta otro cariz a la experiencia de maternidad. Por ejemplo, en algunos casos, la intervención médica es sumamente resistida, pero en otro es considerada una presencia estatal, garante de cuidados sanitarios en contextos de precariedad social mayores. Por tanto, a la hora de pensar si somos vulnerables al parir, es preciso analizar situadamente si hablamos de vulnerabilidades fisiológicas o biológicas, de vulnerabilidades socioculturales y económicas, o de vulnerabilidades creadas por la biomedicina, en su vertiente patriarcal y androcéntrica.

Establecidas las lentes conceptuales, a continuación presento los materiales que conforman el sustento empírico de la tesis a la que referencio en este trabajo, para luego dar paso a ciertos resultados de dicha investigación que sientan bases para la discusión respecto a la lectura a lo Butler de la vulnerabilidad femenina en el parto. 


\section{MATERIALES Y MÉTODOS}

La tesis de referencia analizó diversas instancias de la atención médica del proceso perinatal en la ciudad de La Plata, con técnicas metodológicas complementarias: observaciones con distintos grados de participación en cursos de preparto y salas de espera; entrevistas en profundidad a mujeres-madres, varones-padres y profesionales de la salud; y un extenso corpus de charlas informales y presencias en el campo, desde mayo de 2013 hasta marzo de 2019. Se realizó un estudio de tipo analítico-descriptivo asentado en un abordaje cualitativo, con un diseño de investigación flexible en términos de Valles, (2000) e interactivo (Maxwell, 1996), en tanto que tiene una intención explicativa de los fenómenos sociales en proceso, analizando cómo los actores sociales configuran el marco significativo y simbólico de sus prácticas y representaciones.

Tratándose de una investigación cualitativa, la selección de los casos tuvo como criterio rector la significatividad, la relevancia respecto del tema de investigación y la saturación de la información. En lo concreto, se entrevistaron a 12 profesionales de la salud (gineco-obstetras, parteras, psicólogas, trabajadoras sociales y neonatólogas) que atienden embarazos y partos en instituciones públicas y privadas de salud de la ciudad y asisten partos domiciliarios. En paralelo, se realizaron 7 entrevistas en profundidad a mujeres-madres, siendo tres de ellas en conjunto con sus parejas (varones-padres). El objetivo de estas entrevistas, que en general duraron de promedio una hora con las profesionales y dos horas o más con las mujeres y parejas, fue conocer qué discursos emergen del diálogo, atendiendo y problematizando los sentidos genéricos asignados a la experiencia del PEPP, en particular las vinculadas a los contactos con equipos de salud. En relación con los aspectos éticos, este estudio contó con el consentimiento informado de cada persona indagada y se garantizó la confidencialidad de los datos, usando seudónimos y anonimizando referencias institucionales.

El resultado de la complementariedad de técnicas de investigación permitió contar con un corpus heterogéneo y amplio de registros de discursos, prácticas y experiencias de la atención médica de PEPP en la ciudad de La Plata para el período de estudio (20132019). A los fines de este artículo, me concentré en el análisis de las entrevistas a las mujeres-madres, cuyos perfiles se desglosan en la siguiente tabla. 
Tabla 1: perfiles de entrevistados/as (mujeres-madres y varones-padres)

\begin{tabular}{|c|c|c|c|c|c|}
\hline Entrevista & Fecha & $\begin{array}{c}\text { Propio } \\
\text { nacimiento }\end{array}$ & $\begin{array}{c}\text { Perfil } \\
\text { socioeconómico }\end{array}$ & $\begin{array}{l}\text { Hijo(s)/ tipo (s) de } \\
\text { parto (s) }\end{array}$ & Ámbito \\
\hline $\begin{array}{l}\text { 1. Natalia } \\
\text { (34) y Lito } \\
\text { (36) }\end{array}$ & 25/01/15 & $\begin{array}{l}\text { Cesárea vertical } \\
\text { Clínica privada } \\
\text { «Traumático» }\end{array}$ & $\begin{array}{l}\text { Universitarios; } \\
\text { comerciantes } \\
\text { ligados al turismo }\end{array}$ & $\begin{array}{c}\text { Niña } \\
\text { Parto Vaginal } \\
\text { «Muy lindo» }\end{array}$ & $\begin{array}{l}\text { Clínica } \\
\text { Privada }\end{array}$ \\
\hline \multirow{3}{*}{$\begin{array}{l}\text { 2. Sabrina } \\
\text { (25) }\end{array}$} & \multirow{3}{*}{ 03/02/17 } & \multirow{3}{*}{$\begin{array}{l}\text { Parto vaginal } \\
\text { hospital } \\
\text { privado; } \\
\text { episiotomía y } \\
\text { epidural }\end{array}$} & \multirow{3}{*}{$\begin{array}{l}\text { Ella ama de casa; } \\
\text { el primer padre } \\
\text { albañil; } \\
\text { sin datos de los } \\
\text { otros dos. }\end{array}$} & $\begin{array}{l}\text { Niña A (20 años) } \\
\text { Cesárea } \\
\text { «Traumático» } \\
\end{array}$ & $\begin{array}{l}\text { Hospital } \\
\text { Privado }\end{array}$ \\
\hline & & & & $\begin{array}{c}\text { Niña B (22 años) } \\
\text { Cesárea } \\
\text { «Tranquila» } \\
\end{array}$ & $\begin{array}{l}\text { Hospital } \\
\text { Público }\end{array}$ \\
\hline & & & & $\begin{array}{l}\text { Niño C (24 años) } \\
\text { Cesárea }\end{array}$ & $\begin{array}{l}\text { Hospital } \\
\text { Público }\end{array}$ \\
\hline \multirow{2}{*}{$\begin{array}{l}\text { 3. Valeria } \\
\text { (23) y } \\
\text { Santiago (23) }\end{array}$} & \multirow[t]{2}{*}{$14 / 02 / 17$} & \multirow{2}{*}{$\begin{array}{l}\text { Parto vaginal } \\
\text { «complicado»; } \\
\text { un mes } \\
\text { neonatología }\end{array}$} & \multirow{2}{*}{$\begin{array}{l}\text { Ella ama de casa; } \\
\text { Él empleado en } \\
\text { panadería }\end{array}$} & $\begin{array}{c}\text { Niño A (19 años) } \\
\text { Parto Vaginal } \\
\text { «Caótico» } \\
\end{array}$ & $\begin{array}{l}\text { Hospital } \\
\text { Público }\end{array}$ \\
\hline & & & & $\begin{array}{l}\text { Niño B (23 años) } \\
\text { Parto Vaginal }\end{array}$ & $\begin{array}{l}\text { Hospital } \\
\text { Público }\end{array}$ \\
\hline $\begin{array}{l}\text { 4. Johana (33) } \\
\text { y Damián } \\
(34)\end{array}$ & 4/09/2017 & - & $\begin{array}{l}\text { Universitarios; } \\
\text { profesores. }\end{array}$ & $\begin{array}{c}\text { Niño } \\
\text { Cesárea }\end{array}$ & $\begin{array}{l}\text { Hospital } \\
\text { Privado }\end{array}$ \\
\hline $\begin{array}{l}\text { 5. Mariana } \\
\text { (36) y } \\
\text { Leandro (38) }\end{array}$ & 27/09/2017 & $\begin{array}{l}\text { Parto vaginal } \\
\text { hospital } \\
\text { público; } \\
\text { «lo peor que le } \\
\text { pasó en la vida» }\end{array}$ & $\begin{array}{c}\text { Universitarios; } \\
\text { ambos } \\
\text { bioquímicos }\end{array}$ & $\begin{array}{c}\text { Niña } \\
\text { Parto vaginal }\end{array}$ & $\begin{array}{l}\text { Hospital } \\
\text { Privado }\end{array}$ \\
\hline $\begin{array}{l}\text { 6. Anabel } \\
\text { (27) y Dimitri } \\
\text { (29) }\end{array}$ & 06/03/2019 & $\begin{array}{c}\text { Cesárea } \\
\text { y neonatología } \\
\text { «protocolar» }\end{array}$ & $\begin{array}{l}\text { Emprendedorxs, } \\
\text { trabajos part-time } \\
\text { en negocios } \\
\text { familiares }\end{array}$ & $\begin{array}{c}\text { Niño } \\
\text { Parto vaginal }\end{array}$ & Domicilio. \\
\hline $\begin{array}{l}\text { 7. Emilia () y } \\
\text { Marco () }\end{array}$ & 09/03/2019 & Parto vaginal & Comerciantes & $\begin{array}{c}\text { Niño } \\
\text { Parto Vaginal } \\
\end{array}$ & Domicilio. \\
\hline
\end{tabular}

Fuente: elaboración propia.

\section{RESULTADOS}

Qué fragmentos citar, de un corpus de centenares de páginas de registros de campo y de transcripción de entrevistas, que sean representativos y significativos para el tema del artículo constituye un dilema ineludible. Principalmente porque, durante los años de investigación, la vulnerabilidad en sí no fue una dimensión de análisis, por lo cual lo que presento son algunos resultados y datos que me permiten leer el material empírico en esa clave. Así, en este marco, advertí en el trabajo de reinterpretación del corpus de datos algunos sentidos en que la vulnerabilidad emergió:

a) Vulnerabilidad frente a la decisión médica — pérdida de autonomía—. 
b) Vulnerabilidad potenciada por otras vulnerabilidades asociadas

c) Vulnerabilidad y resistencia, empoderamiento.

Entiendo estas tres formas de expresión de la vulnerabilidad en los PEPP como convivientes y no como excluyentes, ya que considero que, en cada mujer, en cada experiencia situada de proceso perinatal, las referencias se pueden complementar. Para dar cuenta de cómo estas dimensiones emergieron en el campo, retomo algunos fragmentos en los que las entrevistadas narraron cuál era la evaluación que realizaban de su experiencia de parto, siguiendo la línea pensada por Goberna Tricas y Biurrin Garrido, quienes sostienen que

algunas mujeres reconocen que las expectativas forjadas durante el embarazo no coinciden con la realidad en el trabajo de parto. Si la realidad rompe con las expectativas previas o con la decisión previamente manifestada, puede generar cierta frustración y hacer que se sientan vulnerables. (2020, p. 92)

Así, en las evaluaciones requeridas a las mujeres sobre sus experiencias globales de parto, las referencias positivas escasean, contradiciendo o yendo en la dirección opuesta a cierta lectura acrítica que se daba en los relatos. Esto es, mientras que en la mayor parte del relato no mencionaban con desagrado o cuestionamiento muchas de las intervenciones y situaciones que iban narrando, al preguntar por la totalidad del evento, la mayoría de las evaluaciones fueron negativas o, por lo menos, con críticas fundadas. Estas valoraciones negativas suelen descansar en que vinculan su experiencia con su falta de información, autoresponsabilizándose:

Fue cesárea, bueno con ciertas excusas que en ese momento para mi eran muy válidas porque yo no tenía la información que tengo ahora y era muy chica. Me dijeron que era porque estaba de cara y no dilataba y porque durante las contracciones le bajaban los latidos, que de eso yo después me di cuenta que era normal, porque bajaban y volvían a subir. Lo que pienso es que por más que yo en ese momento no lo sabía, me siento una boluda por no haber sabido que me estaban mintiendo en la cara y que el sistema se manejaba de esa manera, y yo con toda mi inocencia de que iban a buscar lo mejor para mí en vez de lo mejor para ellos. Eso es lo que a mí me duele en cuanto a mí misma. Pero yo sé que no fue culpa mía. O sea, aunque yo podría haber estado parada 
de otra manera si hubiera tenido la información. (Sabrina, primer parto, privado)

Cuando terminé de parir dije: «no tengo nunca más un chico si no es cesárea». Así. En el momento que te cae la ficha que estás sola ahí, porque a mí me dejaron sola, dije «nunca más, la próxima pido cesárea. (Mariana, primer parto, privado)

En los testimonios citados de Sabrina y Mariana es evidente cómo la experiencia de vulnerabilidad se asocia al primer tipo que mencioné: vulnerabilidad frente a la decisión médica, quedando sin capacidad ni autonomía para decidir lo que por derecho les corresponde. La relación asimétrica de poder médico-paciente, que posibilita y es germen de la violencia obstétrica, atraviesa la mayoría de las experiencias recabadas en mi tesis —aunque explayarme aquí sobre ello sería imposible.

Esa es mi historia con el nene. O sea, hoy en día no tendría episiotomía, no tendría epidural y no dejaría por ahí tanto manoseo y tanta cosa que, que a mí me, me hacía, la verdad que me hacía sentir re chiquitita, re desprotegida me sentía re desprotegida. Está bien, te genera esa necesidad de estar acompañada un parto, pero me sentí re desprotegida por, primero porque me dijeron «si no te gusta mami... este es un hospital escuela» y eso me hizo sentir desprotegida. (Valeria, primer parto, público)

El caso que narra Valeria da cuenta de una combinación entre el primer tipo de vulnerabilidad y el segundo que propuse, es decir, la interseccionalidad de vulnerabilidades. En este caso, desigualdades de clase y de género combinadas, que hacen que ella deba permitir —o no se pueda negar — la experimentación pedagógica sobre cuerpos de mujeres pobres en hospitales públicos, por el sistema de residencias médicas.

La soledad, la desprotección, las prácticas invasivas, el dolor iatrogénico y la (des)información médica son algunos de los elementos que aparecen en sus relatos. La información emerge como un elemento central de disputa en la atención médica de los PEPP y la posibilidad o el derecho de exigir otra atención como una herramienta desconocida o difícil de ejercer.

Lo que emerge del trabajo de campo es que es posible advertir claves de análisis focalizando en el ámbito de atención — público, privado o domiciliario- en el que 
asistieron los PEPP. Así, uno de los elementos que es posible analizar a partir de los relatos de las entrevistadas es la diversidad de experiencias en los distintos ámbitos de atención. Por ejemplo, la diversidad de tratos en la misma institución, la importancia del derecho a la intimidad, la burocratización y el pago por asistencia, y la (im)posibilidad de parir en casa por cuestiones económicas y de vivienda.

En este marco, primero analizaré algunas particularidades que emergen en la atención en instituciones de salud privadas. En este subsector se dan ciertos privilegios vinculados a, por ejemplo, la exclusividad de la asistencia de un/a profesional (trato personalizado), aunque ese privilegio de clase conlleva muchas veces una contrapartida de dinero:

El tipo [el obstetra] este te cobra un extra por armar el equipo, por atenderte en todo momento, todo. Un extra que nada por lo que es. La atención es sumamente superior a lo que yo vi, al lado mío pasaron embarazadas que las atendieron como vacas, peor [...]. No sé si en todos los médicos es igual, pero este médico me dijo «te cobro un plus para armarte el equipo, no con médicos que te tocan de guardia ni nada». Él te arma un plus el equipo de él. La partera y la pediatra. «Te armo el equipo cuándo sea, cuando esté» y a disposición el día que sea. (Natalia y Lito, primer parto, privado)

Aquí es visible cómo la clase y la disponibilidad de recursos contrarresta ciertas vulnerabilidades a las que se exponen quienes no cuentan con ellos $-\mathrm{y}$ son tratadas «como vacas»—. El cobro de diferenciados no suele ser explicitado durante las consultas prenatales y no suele quedar claro bajo qué conceptos se realiza. Algunas mujeres mencionan que eso cubriría la atención de las parteras, y otras que en realidad lo que se paga es la disponibilidad total del equipo médico. Lo relevante es que a veces dichos equipos suelen mencionar que el plus se cobra para garantizar una atención respetuosa, tal como indica la ley 25.929 de parto respetado. ¿Se paga para que garanticen derechos? ¿El dinero separa a las violentadas — atención por guardia — y a las respetadas —equipo personalizado— dentro de una institución?

En este sentido emerge como clave en los discursos de muchas entrevistadas, por ejemplo, la posibilidad de acceder a habitaciones VIP, en las que no comparten hospedaje posparto con otras mujeres. En este caso hablaríamos de cierto privilegio en la hotelería, pero cuando el dinero hace variar la atención médica, la cuestión toma otro cariz. Asimismo, junto al trato personalizado y la hotelería, la posibilidad que brinda la 
asistencia privada de elegir, se da el privilegio de la gestión de los tiempos, tendientes a reducir las horas de espera.

Al analizar, por otro lado, algunos factores difernciales en la atención pública, bastaría con describir la negativa de la anterior. No hay relación exclusiva con un/a obstetra, no hay posibilidad de cambiar o elegir las condiciones de hotelería ni de gestionar los tiempos de espera. Sin embargo, emergen algunas peculiaridades que son leídas en términos positivos por las mujeres: la gratuidad y el excelente nivel médico, entre otros. El caso de Sabrina es muy interesante porque atravesó el pasaje del sector privado — primer parto — al público — segundo y tercero- - y por ello pudo hacer una lectura comparativa. Se destaca que sus prejuicios o sensaciones se dirigen a la población usuaria del servicio público y no tanto a la atención o a los/as profesionales que allí atienden. De hecho, encuentra en la aparente desventaja de no tener obstetra de cabecera, una oportunidad.

No, en el público te atiende el que está.

¿Y eso te generaba alguna incertidumbre o no?

No, no, me sentía tranquila de que si por ahí un doctor me decía «tal cosa no se puede», por ahí con otro sí se podía. (Sabrina, segundo parto, público)

Junto a pensar las diferencias extrasistemas sanitarios, en el campo emergieron diferencias intrasistemas: Valeria narra diferencias sustanciales entre la atención que recibe en la salita — primer nivel de atención — y en el hospital — segundo nivel—, aunque afirma que: «Yo me siento como en casa en el hospital público, en Maternidad». Por último, en su relato, articula un aspecto diferencial con el tercer ámbito posible de atención: el domicilio:

Mi idea en realidad desde un principio era tenerlo acá en casa, pero sale 15 mil pesos y es una plata que yo tranquilamente... Yo quería bueno eso, lo podría haber juntado por ahí desde un principio, pero me pareció como un gasto... Como si puedo ir y hacerlo gratis, está bien es otra cosa, después me puse a pensar «¿qué hago? Tengo que correr la mesa, está mi abuelo», porque vive mi abuelo acá, «el nene». (Valeria, segundo parto, público)

Si en el análisis de las experiencias de PEPP en los ámbitos de atención institucional se advierten lo que entiendo como dos sentidos de la vulnerabilidad — frente a la decisión médica y en vínculo con otras vulnerabilidades-, al enfocar en la atención no 
institucional emerge entonces el tercer sentido: la vulnerabilidad como resistencia. Si bien los dos primeros sentidos se dan tanto en el sector público como en el privado - ya que en ambos prima la mirada médica, aunque se exprese diferencialmente, $\mathrm{y}$ en ambos es posible la intersección de vulnerabilidades de género, clase, edad, etc.- - el tercero es más visible en el ámbito domiciliario, porque justamente parir extrainstitucionalmente es ya una expresión de empoderamiento.

Una tranquilidad de que iba todo bien o de que efectivamente estaba por nacer, que era lo que yo estaba sintiendo, porque sino era cuánto más va a durar esto. Y nada esos gritos fueron una gran sorpresa para mí también porque... O sea conocer la dimensión de ese poder, viste, muy, o sea nunca en tu vida jamás gritás tanto, entendés. Es la primera vez que gritás (se emociona) [...]. Los pujos son como que vienen solos. Venían solos o sea no era algo que yo decidía pujar, por eso también después de esa experiencia me parece re absurdo eso de «pujá, pujá», viste. Esa figura del que te dice cuándo tenés que pujar, pero bueno igual entiendo que supongo que si te ponen anestesia debe ser diferente [...]. Muy intensa esa fuerza, ese grito [...]. No, no, muy fuerte. Y yo sentía esa cosa que encima que estaba sentada ahí y sentía esa verticalidad, como una fuerza que me bajaba y que me subía a la vez. Y nada y ahí nació. (Ana, primer parto, domicilio).

Esta breve cita de una experiencia de parto domiciliario condensa elementos que permiten preguntarnos si el parto es, efectivamente, el momento de mayor vulnerabilidad de una mujer. $\mathrm{O}$, en el sentido butleriano, a partir de una vulnerabilidad fisiológica, sin negarla, es posible advertir un acto de poder, de resistencia corporal de los más significativos para una mujer.

$\mathrm{Al}$ analizar las experiencias de las mujeres, es importante contextualizarlas en el ámbito de atención, que a la vez suele ser expresión de su perfil socioeconómico, de los partos transmitidos que traen — de hecho, al elegir clínica u hospital mencionan las experiencias de sus conocidas-, de las experiencias que construyen y cómo conciben los PEPP. Esto no quiere decir que se pueda —ni se busque- esencializar las poblaciones usuarias de los distintos ámbitos de atención obstétrica. Se trata de marcar o subrayar algunas regularidades que se corroboran con relatos de partos producidos en los años de trabajo de campo. Al respecto, Gloria Comesaña sostiene que en la atención pública las 
mujeres «pagan con el dolor de sus cuerpos el pertenecer a las clases desposeídas de un país subdesarrollado» (citada por Camacaro Cuevas, 2008, p. 100).

\section{DISCUSIÓN}

La propuesta de este artículo apunta a problematizar cómo «el momento más vulnerable de una mujer» -el parto- puede implicar más vulnerabilidades o, por el contrario, establecerse como un ámbito de resistencia, como Butler señala. Así, emerge la vulnerabilidad como arma de doble filo: nos hace más sumisas, pero allí está el germen de nuestro empoderamiento. Esto es, como mujeres somos vulnerables al control estatal y biomédico al que podemos entender como una vulnerabilidad de género o reproductiva. Y aunque el etiquetamiento como vulnerables sirve al Estado y a la institución médica para promover más y mayor vigilancia y control en pos del cuidado de la reproducción social, la reapropiación de la etiqueta para exigir de qué modos ser cuidadas en una disputa hacia la emancipación reproductiva también es posible.

A través de los fragmentos de entrevistas citados que dan cuenta de las evaluaciones que hacen de sus partos y de las diferencias de atención entre los diversos sistemas de atención sanitaria — público, privado y domiciliario — de un grupo específico de mujeres, quise visibilizar cómo a esta vulnerabilidad reproductiva se suman —o se solapan—otras vulnerabilidades de clase, edad, etnia. No nacen igual quienes nacen en «cuna de oro» que quienes nacen en piso de barro, y no paren igual sus madres. La interseccionalidad de vulnerabilidades — siendo la de género la primera para este caso de estudio- es central como dimensión de estudio de la atención obstétrica en la actualidad.

Siguiendo la argumentación butleriana, entonces, es posible afirmar que las mujeres son objeto de una distribución desigual de vulnerabilidades a la hora de parir, lo que afectará significativamente sus experiencias perinatales. Por efecto de la performatividad de género y médica en la que centralmente nos socializamos sobre cómo ser embarazadas, cómo parir, cómo criar, sujetas a una encerrona de mandatos — provenientes tanto de quienes nos oprimen como de quienes dicen liberarnos—, somos objetos de lo que denominé «vulnerabilidad a la decisión médica». Esta pérdida de autonomía para decidir en el área sexual y (no) reproductiva atraviesa la vida de las mujeres. Junto a esa vulnerabilidad general, existen interseccionalidades de vulnerabilidades, que para el caso 
de estudio es posible advertir en el ámbito de atención y ver cómo la posibilidad —o node elegir dónde parir conlleva determinada vulnerabilidad para la misma experiencia.

Si bien el ideal de la política de humanización de PEPP implicaría que realmente no importe dónde parir, porque se estandarizaría/universalizaría una atención obstétrica respetuosa de los derechos y necesidades de las mujeres, en la actualidad existen diferencias que impactan en las experiencias de las mujeres-madres. Lo distintivo es que, si el sentido común ha establecido que acceder a los privilegios de la atención privada podría garantizar el respeto de los deseos y derechos y buenas experiencias de atención obstétrica, en la práctica hay matices, ventajas y desventajas en los distintos ámbitos. En la tesis pude corroborar que, de manera paradójica, no importa dónde parir: en todos los ámbitos conviven distintos modelos de atención, distintas expresiones de violencia obstétrica, y las relaciones médico-pacientes adquieren características más o menos respetuosas o asimétricas. ${ }^{3}$

Finalmente, y retomando el principal aporte de Butler, encuentro en experiencias de parto domiciliario ${ }^{4}$ expresiones de actos de resistencia a la/s vulnerabilidad/es naturalizadas respecto al momento del parto. En las palabras de la filósofa:

La vulnerabilidad puede ser un momento incipiente y duradero de resistencia. Una vez que entendamos el modo en que la vulnerabilidad entra en la agentividad, nuestra comprensión de ambos términos puede cambiar, y se puede deshacer la oposición binaria entre ellos. Considero que deshacer este binomio es una tarea para el feminismo. (2015, p. 15)

A esta tarea busqué contribuir poniendo en escena la discusión que traje a consideración.

\section{CONCLUSIONES}

A partir del cruce entre una lente conceptual novedosa para entender la vulnerabilidad — compuesta por la mirada clásica que cruza lo antropológico y lo social con la propuesta de Judith Butler que la piensa como el fundamento posible de un acto de resistencia-,

\footnotetext{
${ }^{3}$ Sobre diversas desigualdades que atraviesan la atención médica de los PEP y sus experiencias, he realizado una aproximación en Castrillo (2017).

${ }^{4}$ No quiero sugerir que las mujeres solo pueden resistirse en el ámbito domiciliario — de hecho, he estudiado cómo esta posibilidad de parir en casa emerge como un privilegio cuasielitista - sino interpretar los datos empíricos de la investigación.
} 
analicé experiencias de partos de mujeres platenses. Advertí que en estas experiencias y teniendo en cuenta sus propias evaluaciones de atención en distintos ámbitos —público, privado o domicilio — es posible rastrear tres tipos de vulnerabilidad: a) a la decisión médica — pérdida de autonomía—; b) potenciada por otras vulnerabilidades; y, c) como catalizadora del empoderamiento.

La construcción de la lente conceptual que propuse tuvo como objetivo tensionar la idea naturalizada que sostiene que parir es el momento de mayor vulnerabilidad de una mujer - lo que en general supone pensarla incapacitada, sumisa y pasiva —, para pensar cómo en esa circunstancia, tal vez, se encuentre lo que el activismo feminista viene señalando desde hace años: el momento de mayor poder femenino, que ha incentivado la búsqueda de control de una biomedicina y un Estado patriarcal. De lo que se trata es de deconstruir esta vulnerabilidad construida alrededor de la función esencializada a las mujeres (la reproductiva) y dar cuenta no solo de la posibilidad de empoderamiento, sino las formas que asumen las instituciones para crear y potenciar esas vulnerabilidades para imponer su modo dominante de concebir y abordar médicamente los PEPP. 


\section{BIBLIOGRAFÍA}

BUTLER, Judith. Vulnerabilidad corporal, coalición y la política de la calle. En: Nómadas. 2017, vol. 46, pp. 13-29.

ButLER, Judith. Repensar la vulnerabilidad y la resistencia. En: $X V$ Simposio Internacional de la Asociación Internacional de Filósofas: Filosofía, Conocimiento y Prácticas feministas. Alcalá de Henares: IAPh, 2015, pp. 24-27.

CAmacaro Cuevas, Marbella. Dos sentencias masculinas: parirás con dolor... parirás acostada. En: Delgado de Smith; González (ed.). Mujeres en el mundo. Migración, género, trabajo, historia, arte y política. Valencia (Venezuela): Laboratorio de Investigación en Estudios del Trabajo, 2008.

CASTEL, Robert. De la exclusión como estado a la vulnerabilidad como proceso. En: Archipiélago. 1995, vol. 21, pp. 27-36.

CASTRILlO, Belén. Hacer partos y parir: hacia una sociología de la atención médica de embarazos y partos. [Tesis doctoral]. Universidad Nacional de La Plata, 2019. Disponible en: http://sedici.unlp.edu.ar/handle/10915/78629

CASTRILlo, Belén. "YYo no puedo parir en casa porque tengo piso de tierra'. Desigualdades sociales en la atención médica de embarazos y partos, y en las demandas políticas por el parto respetado». [Ponencia] IV Seminario Internacional Desigualdad y Movilidad Social en América Latina. La Plata, junio de 2017.

Esteban, Mari Luz. El género como categoría analítica. Revisiones y aplicaciones a la salud. En: Cuadernos de psiquiatría comunitaria. 2003, vol. 3, núm. 1, pp. 22-39.

FEITO, Laura. Vulnerabilidad. En: Anales del sistema sanitario de Navarra; 2007, vol. 30, pp. 7-22.

Goberna-Tricas, Josefina; Biurrun-Garrido, Ainoa. El alivio del dolor en el parto. Empoderamiento y vulnerabilidad de las mujeres en la toma de decisiones. Estudio cualitativo. En: MUSAS. Revista de Investigación en Mujer, Salud y Sociedad. 2020, vol. 5, núm. 1, pp. 79-97.

MaXwelL, Joseph. Qualitative Research Design. An Interactive Approach. Thousand Oaks: Sage, 1996

VAlles, Miguel. Técnicas cualitativas de investigación social. Madrid: Síntesis, 2000. VALLs LloBet, Carme. Mujeres, salud y poder. Madrid: Cátedra, 2009. 